Paediatrica Indonesiama $15: 93$ - 104. March - April $19 \% 5$.

** WHO Consultant on Dengue Hemornhagic Fever, Children Hospital, Bangkok, Thailand

\title{
Dengue Hemorrhagic Fever: Problems and Progress*
}

$$
\text { by }
$$

\section{SUCHITRA NIMMANNITYA **}

\begin{abstract}
The paper nepresients a clinical reappraisal of idengtue hemorrhagic fever ( $D H F$ ) and the anuthor's purpose to briefly review and discuss the pertinent datia obtainjed to diate. The mast frighteming pictume of DHF that clearly diffienentiates this new climiaal entity from the classical dengue illness and contribute to its cause of death is the shock syndrome.

The major pathophysiolagic chatonge that leads to hypovolemia and subsequent shock is the ?eakiagre of plasma. The acute anset of shock and the rapid, often dnamatic clinical recovery when treatied, togrether with the fact that no destructive or inflammatomy vascular lesion has been observed suggested a functional vascular permaability change possibly isue to a short racting pharmacological mellikator.

Epidemiological and serologiaal observations circumstiantiated a hypothesis that DHF occurs as a result of a second infection with a heterologous diengnue vimus. Rlecent stiudies strongly impliaated an immunalogical mechanism involving activation of complemient system and its proaiucts, complemient-iderived amaphylatoxins as nesponsible for the imitiation of shock. The findings of low fibrinagien and appreamance of fribrinogen split product in correlation to the diseadse severity together with the constamtly found thrombocytopemia indicated occunence of comsumptive coagrulopathy but probably mot a major pathogenic role: The fract that in most cases, jarly and effective neplaciement of plasma volume mesults in favounabile outcome, and that sigmificant bileedings when presemt, usually occur after the onsiet of shock, and support to the major paithagemic rols. of the increased vascutar puermalability and extnavasiation of plasma.
\end{abstract}

- Invited Paper at the Third National Indonesian Paediatric Congress, Surabaya, July 1-6, 1974. Received August 12, 1974. 
The year 1956 was important in the hilstory of dengue and perhaps in the history of viral diseases in generall for it marked the first recognition of dengue hemorrhagic fever (DHE), a new urban disease of children characterized by fever, hemorrheric diathesis, henatomegaly and a life threatening shock syndrome. After the firet epidemic in Manila the disease has spread to other counkries in the Southeast Asia and the Western Pacific regions where Aedes aegypti mosquitoes (a dengue vector) are abundant.

In some of these areas during DIF outbreaks chikungunya vimus, also transmutted by Ae. aegypti, sometimes concer simitar but milder disease, more like classical dengue fever (Nimmannitya et al., 1969). Only dengue viruses are responsible for the severe life threatening diseases which is now considened as of significance among communicable diseases in most tropical Astian countries. The repetition of outbreaks or their extension to new areas have raised during the past two decades a number of public health problems which are complicated, difficulit and not yet completeily solved.

DHF is considerably a new disease or a new variant of dengue infection because of its unusual hemorrhages and the shock syndrome so-called dengue shock syndrome (DSS) which has been known for more than a century in Asia are largely age dependent, the disease is mild in children and more severe in adulits. Infants and young children with dengue infection have syndrome ranging from undifferentiated fever to mild febrile illness sometimes with the triad of high fever, pain in various parts of the body and rash. The diverse is known as non-fatal, death is racher an exceptional. DHE on the contrary, attacks mostly children under age 14 years and caused significant mortality among preschool age children of Southeast Asia and the Western Pacific regions where classical dengue syndrome is a rare incidence among indigenous people.

During the past two decades several research approaches have produced information which contributed to our present understanding of cpidemiology, virology, immunology and clinical research. In each of these fiellds, there are significant unanswered questions remaining to be further pursued. In the point of view that some of the existing problems can be solved by appropriate action in applying the knowledgeg we already possess, it is my purpose to briefly review and discuss the pertinent data obtained to date on some aspects of DHF.

\section{Virological and Epidemiological Aspects}

The four dengue viruses form a subgroup of the group $B$ arbovirus based on close antigenic relationships due to common or cross-reacting antigenic determinants present in 
FIG. I : Graph showing age distribution.

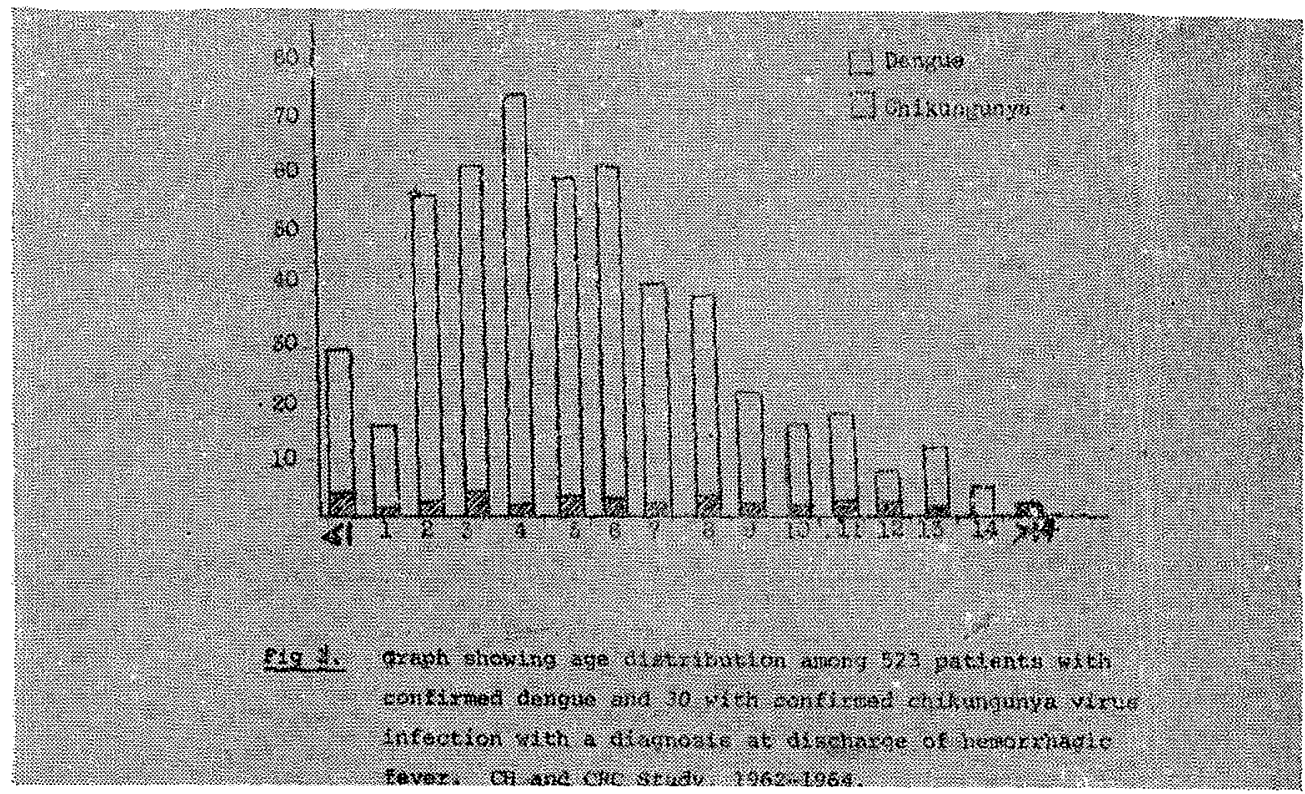

FIG. 3 : The Spectrum of Dengue Diseases.

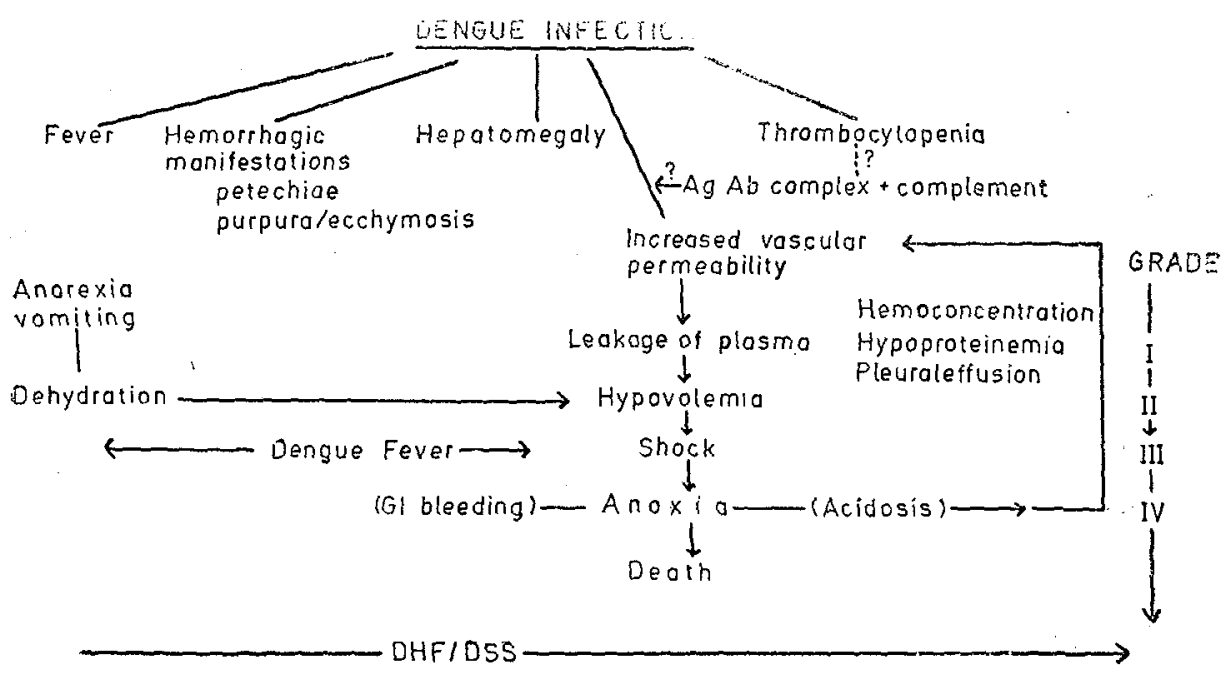


FIG. 2 : Age distribution and accurence of shock in patients with primary and secondary dengue antibody response and in 452 DHF patients a secondary dengue antibody response. (Modified from Halsteed Nimmannitya, \& Cohen).
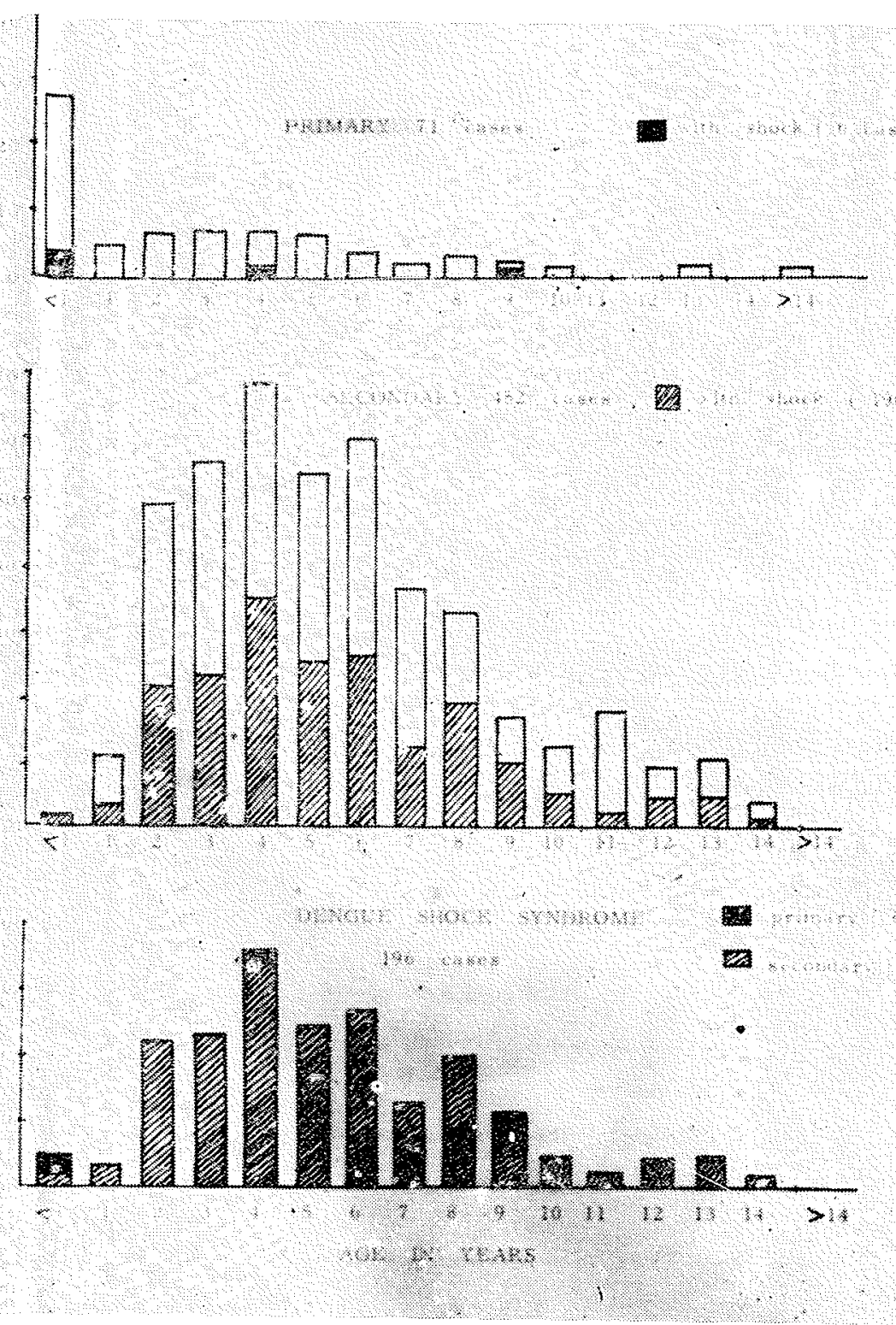
complaint shortly before onset of shock.

Shock is characterized by a rapid and weak pulse with narrowing of the pulse presisure $(20 \mathrm{~mm}$. $\mathrm{Hg}$. or less, regardless of the pressure levels, e.g. 100/90 mm. Hg.) or hypotension, with cold, clammy skin and restlessness, the onseit of shock is usually abrupt and often profound. The patients are in danger of dying if appropriate treatment is not prompitly given. The duraition of shock is short, the patient may die within $12-24$ hours or recover rapidly following appropriate antishock therapy. Prolonged uncontrolled shock may give rise to a more compllicated course with metabolic acidosis, severe gastrointestinal hemorrhage and a poor prognosis.

Thrombocytopenia and concurrent hemoconcentration are constant findings. A platelet count of below $100,000 / \mathrm{mm}^{3}$ is usually found between the third and eight days, the platelet count falls and hematocrit rises before onset of shock.

The cllinical diagnosis based on four major clinical manifestations:

a. fever, high continuous for $2-7$ days.

b. hemorrhagic manifestations including at least a positive tourniquet test with any other.

c. hepartomegaly.

d. circulatory disturbances - in the form of shock in severe cases. And the two constant laboratory findings thrombocytopenia and concurrent hemoconcentration provide the basis for accurate and rapid diagnosis before shock or irreversible shock occurred.

The spectrum of DHF/DSS is classified according to disease severity into four grades (Nimmannitya et al., 1969).

Grade I. Fever accompanied by non-speciffic constitutional symptoms, the onily hemorrhagic manifestation is a positive tourniquet itest.

Grade II. The addilitional manifestation to those of grade $I$ is spontaneous bleeding; skin and/or other hemorrhages.

Grade III. Circullatory failure manifested by rapid and weak pulse, narrowing of pulse pressure $(20 \mathrm{~mm}$. $\mathrm{Hg}$. or less) or hypotension, with the presence of cold clammy skin and restlessness.

Grade IV. Profound shock with undetectable blood pressure and pulse.

Table I shows the occurence of major manifestations and laboratory findings in relation to disease severity. The presence of ithrombocytopenia with concurrent hemoconcentration will differentiate grade I and II, non shock cases of DHF from classical dengue fever, and from other virall infections.

\section{Pathophysiology}

\section{Vascular permenubility}

The major pathophysiological abnormality seen in DHF/DSS is an 
acute increase in vascular permeability that leads to leakage of plasma. Plasma volume studies revealed a re duction of more than $20 \%$ in severe cases (Suwanik et al., 1967) supporting evidence of plasma leakage, include hemoconcentration, hypoproteinemia, serous effusion found at postmortem, and pleural effusion found on X-rays.

In severe cases, the onset of shock is acute, hematocrit rises sharply as plasma escapes through the endothelium. Hypovolemic shock, as a consequence of critical plasma loss leads to tissue anoxia, metabolic acidosis and death if uncorrected.

In most cases, early and effective replacement of lost plasma with plasma, plasma expander and/or fluid and electrolyce solution results in a favourable outcone. The acute onset of shock, and the rapid, often dramatic clinical recovery, together with the fact that no destructive or inflammatory vascular lesions are observed (Bhamarapravati et al., 1966) suggest transient functional vascular changes possibly due to a short acting pharmacological mediator (Russel, 1970).

Electrolyce disturbance frequently found and is considerably significant in the treatment is hyponatremia. This can be attributed to salt depletion in excess of water; salt depletion may have been present to some degree due to reduced salt intake from anorexia and increased loss from vomiting and excessive swe- ating, water retention is the result of both decreased renal excretion and increased oxidative water formation from increased metabolism during the febrile stage. Few cases have evidences suggesting inappropriate $\mathrm{ADH}$ secretion (Varavithya et al., 1973).

Early in the course of illness respiratory alkalosis with mild metabolic acidosis may be found, but in the cases with prolonged uncontrolled shock metabolic acidosis is more common and may cause a more complicated course if not corrected.

\section{Hemollhagic Diathesis}

Bleeding manifestations in DHF range from mild skin hemorrhage as positive tourniquet test and petechiae, epistaxis, gum bleeding, to the severe sometimes fatal-massive gastrointestinal! hemorrhages.

\section{Hematologic changes in DHF}

\section{Vasculopathy}

- Capillary fragility changes result in positive tourniquet test.

- Capillary response to injury is poor as manifested by the prolonged bleeding time.

\section{Thrombocytopenia}

Thrombocytopenia is a constant finding in DHF. Platelet counts fall to a moderately or markedly low level by day 3, usually preceeding shock in severe cases and rise to normal or sometimes supernormal level by day 8 to 10 . It is important 
to note that although the level of low platelet counits is in correlation with the disease sevenity, the very low level of platelets (below 40,000 per cu. mm.) is not always correlated with severe bleeding. The reverse, however, is always true, i.e. in cases with severe hemorrhage the platelet counits are always very llow.

Bone marrow studies in these patients indicated hypocellular and decreased number of megakaryocytes suggesting decreased production during the early febrile phase. This is probably one factor - a minor one - in explaining thrombocytopenia in DHF (WHO, 1966).

The rapid fall of platelet counts to a very low level polinits ito a destructive process or sequestration as a more important rolle. The pllatelets survival studies in some of these patients revealled an unusuall shortage of its half life implicating enhanced destruction of the circulating platelets (Mitrakul, 1973). A consumptive coagullopathy is one possibillity, but is not absolute evidence of platelets destruction, just as a low platelet count is no proof of initravascular coagulation.

The observation of sudden drop of platelets concurently with hemoconcenitration preceeding shock as shown in Fig. 4, occurred simuiltaneously with the rising antidengue antilbody suggests another possibility that platelets may be aggregated or adhered to antigien antibody complex.

\section{Coagulopiathy}

Table 2 shows the results of fibrinogen, fibrin degradation product (FDP) and platelets in correlation to the disease severity. 'The low level of fibrinogen and the presence of FDP together with the constantly found thrombocytopenia could be interpreted as evidence of disseminated intravascular coagulation (DIC). (WHO, 1973).

The screening coagulogram in some of these pativents with high FDP level revealled prolonged partial thromboplastiln time (PTT) in $54.6 \%$, prolonged prothrombin time (PT) in $33.3 \%$ and normal thrombin time. These findings together with the observation that FDP is not always found in severe cases, and when present, the llevel is rather low, particularly if compared to the level found lin classical DIC due to gram negative septicemia (Suvatite et al., 1973), suggest that mill consumptive coagulopaithy may take place in DHF but no frank DIC which plays a significant pathogenetic role.

The assay of clotting factors revealed mild to moderate reduction of factors II, V, VII, IX, X (Weiss and Halstead, 1965; WHO, 1966) and also factor XII (Mitirakul, 1973; Edelman et al., 1973), in severe cases with shock whille the non shock cases always showed normal coagulograms. In case of shock with severe bleeding there was a definite reduction in all coagulation factors except 
factor VIII. The normal level of factor VIII, although no absolute evidence againsit intravascular coagulation, does, however, cast some doubt on the occurrence of consumptive coagulopathy in DHF.

It should also be noted that hepatomegaly is a constant finding in $\mathrm{DHF}$, and that the liver function profile showed mild to moderate elevation of serum transaminases; at post mortem there were always changes indicating some degree of liver damage. It is quite possible that in severe cases of DHF, a certain degree of liver involvement may cause coagulation defects.

It thus appears that skin bleeding in DHF in general is due to capillary factors and thrombocytopenia in some extent. The gross massive bleeding is possibly due to more complex mechanisms; thrombocytopenia, coagulation defects, and probably in some extent due to consumptive coagulopathy particularly in those who have been in prolonged incontrolled shock with complicated metabolic acidosis. Further information is needed on the role of shock per se in causing or potentiating DIC.

\section{Pathogenesis}

The association of secondary infections and the consequent anamnestic antibody response with DHF is firmly estallished. This indicates that an immunological mechanism is involved. The complement studies car- ried out in recent years have greatly clarified the pathogenetic mechanisms in DHF and DSS. The important central role of complement activation and the consumption of $\mathrm{C} 3$ have been conclusively demonstrated (WHO, 1973; Bokisch et al., 1963).

The occurrence of immune comploxes is presumed on the basis of aimultaneous presence of virus and antibody in patient's serum, and this is supported by evidence of C1 consumpition and the finding of $I g G$, dengue antigen, and C3 deposited in kidneys of patients convalescent from DHF (Futrakul et al., 1973).

Recent studies revealed that immunological enhancement of dengue virus replication in peripheral leukocytes in the sense that viremia in secondary infection is quanititatively greater than viremia in primary infection (Marchetitee, 1974). These findings suggest that cell mediated immunity may also be involved in the pathogeneitic mechanism of DHF. In secondary infection with enhanced virus replication, and the rapild rise to a high level of andidengue IgG antibody the interaction to form immune complex is more feasible and become significant.

Fig. 5 shows the complement profilles of DHF patients (WHO, 1973), complement depression in DHF involved primarily C3, C3 preactivator (C3PA), C4, and C5. The marked depression of serum level of comple 
ment was found to be correlated with the severity of the disease.

The activation of $\mathrm{C} 3$ and $\mathrm{C} 5$ is accompanied by dissociation of low molecular weight peptides called C3a and C5a anaphylatoxins which are very potent permeability increasing factor and have the capabillity ito release histamine (Muller-Eberhard and Vollota, 1971). Since the C3 concentration was reduced by at least $33 \%$ in the shock patients and a reduction of $33 \%$ in the C5 levels also occurred in $89 \%$ of such patients, it may be that large amounts of anaphylatoxin: are liberated during the shock phase. Although plasma contains a powerful inactivator of the two peptidles (Bokisch and Muiler Eberhard, 1971), it is quite possible that these peptides contribute to the development of shock before they undergo inactivations (WHO, 1973). The fact that anaphylatoxin is rapidly inactivated and eliminated from the circulation is in accordance with the short period and often dramatic recovery of shock when treated.

The question of a possible rolle of bradykinin in causing permeability increase and hypovoliemila has been clarified in the recent studies which showed no gignificant change of bradykinin or kallikrein inhibitor in relation to the disease severity. This negative finding in kinin study together with the evidence of rapid fall of C3 preceeding shock, during shock and the return to nor- mal in convalescence observed in the same studies give strong support to the central role of complement activation in the pathogenesis of DHF (Edelman et al., 1973).

The complement has been shown to be capable of initiating blood coagulation through the platelets (Zimmerman and Muller-Eberhard, 1971).

It is quite possible thiat the mild consumptive coagulopathy observed in $\mathrm{DHF}$ is a consequence of complement activation occurring during its intermediate pathway and probably does not play a major pathogenic role. This iis supported by the fact that significant bleeding is infrequent in DHF, when present usually occurs after the onset of shock, after a prolonged uncontrolled shock.

The evidences that in most cases early and effective replacement of plasma volume results in favourable outcome and that with early treatment of shock the incidence of severe bleeding is further reduced, strongly indicate the major role of the increased vascular permeabilitity and extravasation of plasma, in the pathogenesis of DHF/DSS.

\section{Treatment}

As in other virral infections there is mo specific antiviral agent for dengue infection. Symptomatic and supportive measures are, however, effective in DHF and DSS providing prompt and appropriate antishock 
therapy is given before itrreversible shock takes place.

DSS is a hypovolemic shock due to lealkage of plasma, hyponatremia is commonly found, thus immediate adminilstration of intravenous fluid to expand plasma volume is most essential. Serial hematocrit determinations are most essential guide in therapy since they reflect the degree of plasma leakage and the need for intravenous fluid therapy. Hemoconcentration usually precedes blood pressure and pulse changes. Moreover the hematocrit is also a good indication for stopping the intravenous fluid when the reabsorption of the extravasated plasma takes place.

The experience in the management of shock in DHF at the Children's Hospital, Bangkok, has proved satisfactorilly with the following regi mens:

1. Immediate replacement of the existing plasma loss with plasma or plasma expander, and/or isotonic salt solution.

2. Correction of further plasma loss and maintaining of circulating volume for another 12 to 24 hours or at the most 48 hours.

3. Correction of ellectrolyte disturbance and/or acid base imbalance.

4. Fresh blood transfusion in case of massive bleeding.

The steadily decline in mortality rate after 1964 withour using steroids (Fig. 6) precludes the necessity of this drug in the therapy of DHF/ DSS. Early recognition of cases as well as early and appropriate treatment of shock with proper monitoring of the patient's conditions play important roles in the reduced mortality rate from $9 \%$ in 1964 to $2 \%$ in 1973.

A controlled study comparing the results of treatment of DSS with and without steroids showed no difference between the two methods (Pongpanich et al., 1973). The most recent blood cortisol level studies in DHF patients showed high cortisol level during the stage of shock. The level in shock cases is 4 to 5 times higher than in control (Tuchinda et al., 1974). This could be simply interpreted as no evidence of adrenal insufficiency. Nonetheless the possibility of harm effect of the high cortisol levels seen in the severe cases should not be overlooked. A further investigation is needed.

In conclusion, in spite of the many questions and puzzles that remain, body of knowledges in various aspects of DHF has compiled. Better understanding of the disease improves clinical diagnosis and management of $D H F$, and the mortality rate reduces. Good and accurate clinical criteria for diagnosis reduce the problem of overdiagnosis and a great demand of hospital beds. Early recognition of cases is also important in surveillance program of DHF, and for the control program. 
In view of the fact that there is no vaccine availlable, the fundamental measure to be taken in the disease prevention and control is to conitrol the vector mosquitoes. To achieve the goal in DHF control there is a need for close cooperation among all concerned. A surveillance program for DHF at the international as well as at local levels is most desirable. Logistics of the operattion and research on various aspects of DHF may vary from one country to another. However, the experiences gained in one country may interest the others.

\section{REFERENCES}

1. BHAMARAPRAVATI, N., BOONYAPAKNAVIK, V., and NIMSOMBURANA, $P_{\text {: }}$ : Patholiogy of Thai hemorrhagic fever. An autopsy study. Bull. WHO $35: 47-48$ (1966).

2. BOKISCH, V.A. and MULLEREBERHARD, H.J. : J. Clinical Invest. $49: 2427-2436$ (1971).

3. BOKISCH, V.A., TOP, F.H. PONGPANICH, B., PANCHAPAKARN, P. and NIMMANNITYA, $\mathrm{S}$. : Catabolic rate of $\mathbf{C} 3$ and $\mathbf{C 1}$ complement in dengue hemorrhagic fever patients (19721973). The SEATO Medical Research Lab., Bangkok, The Annuall Progress Report (1973).

4. EDELMAN, R, NIMMANNITYA, S., COLMAN, R.W. TALMO, R.C. and TOP, F.H. : Evaluation of plasma kynin system in dengue hemorrhagic fever (in press, 1973).

5. FISCHER, D.B. and HALSTEAD, S. B. : Observations related to the pathogenesis of dengue hemorrhagic fever. Examination of age specific sequential infection rate using a mathematical model. Yale J. Biol Med. 42 : 329 (1970).

6. FUTRAKUL, P. et al. : Renal involvement and reticuloendothelial-system clearance in dengue hemorrhagic fe- ven. J. med. Ass, Thailand $56: 33-39$ (1973).

7. HALSTEAD, S. B., NIMMANNITYA, S. and OHEN, S.N., : Observation related to a pathogenesis of DHF. Yalo J. Biol. Med. $42^{\prime}$ : 311-328 (970).

8. HALSTEAD, S. B., NIMMANNITYA, S. YAMARAT, C. and RUSSEL, P.K. : Hemorrhagic fever in Thailand. Newer knowledge regarding etiology. Jap. J. Med. Sci. Biol. 20 : 96-103 (1967).

9. IN : REPORT OF AN INTERNATIONAL COLLABORATIVE STUDY: Pathogenetic mechanisms in DHF. Bull. W.H.O. 48 : 117-133 (1973).

10. IN : WHO INTERREGIONAL SEMINAR ON MOSQUITO-BORNE HEMORRHAGIC FEVER OF SOUTH AND SOUTH-EAST ASIA. Bull. W.H.O. 35 (1966).

11. MARCHETTE, N.J. : Immune enhancement of dengue virus replication in peripheral leucocytes - a possible me. chanism in the pathogenesis of dengue shock syndrome. In : paper presented at the 13th SEAMO-TROPMED Seminar, Saigon (1974).

12. MITRAKUL, C. : Hematologic aspects in DHF. Chulalongkorn med. J. Thailand 17 : 203-207 (1973). 
33. MULLER-EBERHARD, H.J. and VOLLOTTA, E.R. : Formation and inactivation of anaphylotoxins. In : Froceedings of the 2nd Int. Symp. on the Biochemistry of the acute allergic Reaction. Quoted in Bull. W.H.O. $48: 131$ (1973).

14. NIMMANNITYA, S. HALSTEAD, S.B., COHEN, S.B, and MARGIOTTA, M.R. : Dengue and chikungunya virus infection in man in Thailand, 19621964. Amer. I. trop. Med. Hyg. 18 : 954-971 (1969).

15. PONGPANICH, B., BHANCHET, P., PANTCHAYAKARN, P. and VALYASEVI, A. : Studies on DHF; clinical study, an evaluation of steroids as $\mathbf{a}$ treatment. J. med. Ass. Thailand, 56 : 6-14 (1973).

16. PUSSEL, P.K. : Pathogenesis of dengue shock syndrome : evidence for immunological mechanism. In Proc.6th int. Symp. on Immunopathology pp. 426-435 (Grindelwald, Basel Schwabe, Switzeriand 1970).

17. RUSSEL, P.K. and NISALAK, A. : Dengue virus identification by the plaque reduction neutralization test. J. Immunol. 99 : 291 (1967).

18. SABIN, A.B.: Research on Dengue during World War II. Amer. J. trop. Med. 1: 30 (1952).

19. SUVATTE, $V$, et al : Studies en serum complement $\mathrm{C} 3$ and fibrin de- gradation products in Thai Hemorrhagic fever. J. med. Ass. Thailand 56 : 24.32 (1973).

20. SUWANIK. $\mathrm{R}$, et al : Plasma volume and other fluid spaces studies in Thai H.F.J. med. Ass. Thailand, 50: 48-66 (1967).

21. TUCHINDA, C., VISUTAKUL, P., ANGSUSINGHA, K. and PUNNAKANTA, L. : Blood cortisoli level in Thai hemorrhagic fever. In preparation (1974).

22. VARAVITHLA. W. et al: Studies on DHF; Electrolyte Study. J. med. Ass. Thailand $56: 15-23$ (1973)

23. WEISS, H.J. and HALSTEAD, S.B.: Studies of hemostasis in Thai hemoryhagic fever. J. Pediat. 66: 918-926 (1965).

24. WHITEHEAD, R.H., CHICUMPA, V. OLSON, L.C. and RUSSEL, P.K. : Sequential dengue virus infections in the white-handed gibbon. Amer. J. trop. Med. Hyg. 19: 94 (1970).

25. WINTER, P.E. et al : Recurrenre of epidemic DHF in in an insular setting. Amer. J. trop. Med. Hyg. $18: 573$ (1969).

23. ZIMMERMAN, T.S. and MULLEREBERMARD, H.J. : J. exp. Med. 134. 1601-1607 (1971). Quoted in Bull. W. H.O. 48 : 131 (1973). 